Historic, Archive Document

Do not assume content reflects current scientific knowledge, policies, or practices. 


\section{Stark Bros.}

\section{Nurseries \& Orchards Co.}

General Offices

Louisiana, Missouri

BRANCH NURSERIES; Dansville, N. Y.; North Girard, Pa.; Huntsville, Ala.; Fayetteville, Ark.; Marionville, Mo.; and other points.

(Communications should be addressed to headquarters-Louisiana, Mo., where all correspondence is handled.)

\section{Important---Read Carefully}

We pay freight to any railroad station in the United States on orders to the amount of $\$$ ro.oo net, provided one-fourth or more cash accompanies the order. For all cash before shipment $10 \%$ discount will be allowed from prices quoted herewith.

We box and pack free.

All boxes are lined with paper-a safeguard against both drying out and freezing.

We absolutely guarantee safe arrival.

Orders lost or spoiled in transit we refill free.

We use the utmost care to avoid all mistakes and Stark Trees may be depended upon to bear fruit true to label, but if through error or otherwise any should prove untrue, we will replace free of charge, but are liable no further. Our just and liberal dealing since 1816 is our strongest guarantee to customers.

Entomologist's certificates on all shipments.

SPECIAL. Will make special prices on large orders. Write for estimate.

One-year trees are graded in two sizes-3 to $5 \mathrm{ft}$. and 2 to 3 $\mathrm{ft}$. Each size graded strong; our 2 to $3 \mathrm{ft}$. size equals the largest size of many nurseries.

Two-year trees are graded into three sizes. $\mathrm{XXX}$ is the size sure to please. XX size excels "first-class" of some nurseries. No second-class defective or cull trees go into any size, but to the brush pile.

BY PARCEL POST. Fruit trees not larger than 2 to 3 ft. mailed prepaid for $3 \mathrm{c}$ per tree above prices of smallest grade listed. Grape, berries, currant at $2 \frac{1}{2} \mathrm{C}$ extra per plant.

SPECIAL SERVICE DEPARTMENT. For information on subjects relating to fruit culture and selection of varieties for given localities, cultivation, spraying, etc., you a re referred to our Special Service Department which is in charge of trained, experienced men. The services of this department are free, and we invite correspondence.

SPECIAL NOTICE. When buying apple, pear, peach, quince, cherry, crab apple, nectarine, plum, apricot, dwarf pear and dwarf apple, the following explanation of Price List should be read carefully.

rooo Rate- 300 (or more) of a kind (as 300 apple, 300 pear) but not less than ro of a variety.

roo Rate-3o (or more) trees of a kind (as 30 apple, 30 cherry), but no less than ro of a variety.

Io Rate-1o or more of a variety, but if as many as 30 or more, customer is entitled to roo rate.

Each Rate-Less than ro of any variety at Each rate. 


\section{Apple}

Commercial Varieties

TWO-YEAR, XXX, 5 to $7 \mathrm{ft} \ldots . .40$

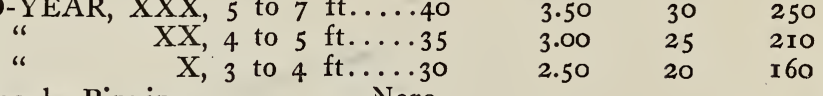

Albemarle Pippin

Nero

Alexander

Arkansas Black

Banana (Winter)

Newtown Pippin

Black Ben (Trade-Mark)

North Western Greening

Ortley (White Bellflower)

Champion (Trade-Mark) Paragon Winesap

Delicious (T.-M.) S. Delicious Rome Beauty

Duchess (Oldenburg)

Early Melon

Early Ripe

Fall Pippin

Gilbert Winesap

Gravenstein

Grimes Golden

Ingram

Jefferis

Jonathan

Kinnaird Choice

Senator (Trade-Mark)

Spitzenburg

Stark Delicious (Trade-Mark)

Stark King David (T.-M.)

Stayman Winesap.

Steele Red (Red Canada)

Sweet Bough

Vandiver Improved

Virginia Beauty

Wagener

Wealthy

King David (T.-M.) S K. D. Western Beauty (Ohio Nonp.)

Liveland Raspberry White Winter Pearmain

Magnet (Magnate) Winesap

Malinda Windsor

M. B. Twig (Stark Strain) Yellow Transparent

McIntosh Red

Minkler Improved

York Imperial

\begin{tabular}{|c|c|c|c|c|}
\hline Usual Varieties & Each & & & 1000 \\
\hline ONE-YEAR, 3 to $5 \mathrm{ft} \ldots \ldots$ & $\cdots \cdot 30 c$ & $\$ 2.60$ & $\$ 22$ & $\$ 180$ \\
\hline " $\quad 2$ to $3 \mathrm{ft} \ldots \ldots$ & $\ldots .25$ & 2.10 & I7 & 130 \\
\hline TWO-YEAR, XXX, 5 to $7 \mathrm{ft}$ & $\cdots \cdot 35$ & 3.10 & 27 & 230 \\
\hline " $\mathrm{XX}, 4$ to $5 \mathrm{ft}$ & $\cdots \cdot 30$ & 2.60 & 22 & 190 \\
\hline $\mathrm{X} 3$ to $4 \mathrm{ft}$ & $\ldots \ldots 25$ & 2.10 & & 140 \\
\hline Akin & Lowell & Orange & ippin & \\
\hline American Summer Pearmain & Maiden & lush & & \\
\hline American Golden Russet & McMaho & White & & \\
\hline Baldwin & Northerr & Spy & & \\
\hline Bayard & Old Wif & Pippin & & \\
\hline Bellflower, Yellow & Rambo & & & \\
\hline Benoni & Red Ast & chan & & \\
\hline Bonum & Red Jun & & & \\
\hline Charlamof (Early Duchess) & R. I. Gr & ening & & \\
\hline Chenango Strawberry & Roman & $\mathrm{em}$ & & \\
\hline Cox Orange & Roxbury & Russet & & \\
\hline Early Colton & Salome & & & \\
\hline Early Harvest & Stark & & & \\
\hline Fameuse (Snow) & Shockley & & & \\
\hline Fanny & Sutton B & auty & & \\
\hline Hubbardston Nonsuch & Talman & weet & & \\
\hline Hyde King (King of the West) & William & Favori & & \\
\hline Jeniton & Willow & wig & & \\
\hline King Tompkins Co. & Wolf $\mathrm{Ri}$ & & & \\
\hline Iady Sweet & Yates & & & \\
\hline Longfield & Yellow & orse & & \\
\hline
\end{tabular}

\section{Special Varieties}

ONE-YEAR, 3 to $5 \mathrm{ft} \ldots \ldots \ldots \ldots 40 \mathrm{C}$

$$
\text { " } 2 \text { to } 3 \mathrm{ft} \ldots \ldots \ldots \ldots 35
$$

TWO-YEAR, XXX, 5 to $7 \mathrm{ft} . \ldots 45$

" XX, + to $5 \mathrm{ft} \ldots .40$

$\mathrm{X}, 3$ to $4 \mathrm{ft} . \ldots 35$

IO

I00 1000

$\$ 3.50 \quad \$ 30 \quad \$ 250$

$3.00 \quad 25 \quad 200$

$4.00 \quad 35 \quad 300$

$3.50 \quad 30 \quad 260$

$3.00 \quad 25 \quad 210$

All Summer Bellflower Imp'd (M. Orange) Bismarck

(Special Varieties Apples Continued Next Page.) 


\section{Apple---Continued}

Chicago (Trade Mark)

Colorado Orange

Dudley

Ensee

Estelline

Fall Winesap

Giant Jeniton

Gravenstein, Banks Red

Gravenstein, Red; Stark Strain

Hackworth

Henry Clay (Trade Mark)

Imperial Rambo

Lady Apple

Lansingburg (Rock Pippin)

Lowry (Dixie of Virginia)

Magoon

Mammoth Grimes Golden

Mother (A Fall Delicious)

New Zealand Northern Spy
Okabena

Primate

Patten Greening

Peerless

Red Jefferis

Red Rome Beauty, Illinois

" Rome Bty, Ohio Bright

“ Rome Bty, Ohio Dark

" Rome Beauty, Washington

Scarlet Pippin

Shannon Imp'd

Stark-Star (Trade-Mark)

Stark Summer Queen

Summer Champion

White Pippin (Stark Strain)

Wilson Red June

Winter Gravenstein

Winter Maiden Blush

Wismer Dessert

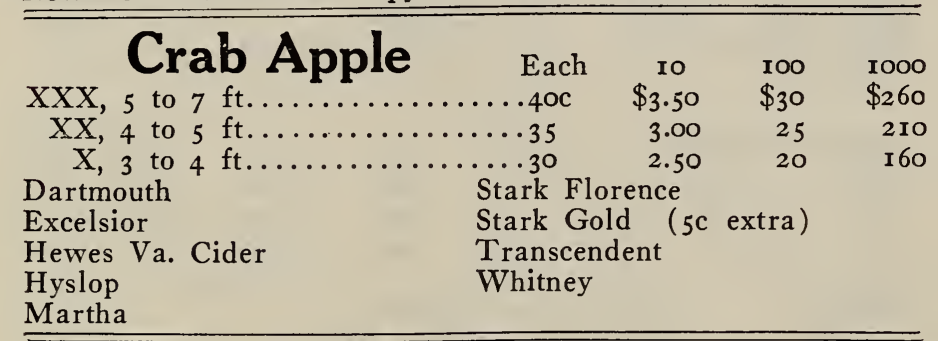

\begin{tabular}{|c|c|c|c|c|}
\hline 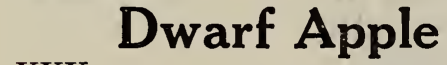 & & Io & 100 & 100 \\
\hline $\mathrm{XXX}$ & .6 & $\$ 6.00$ & $\$ 55$ & $\$ 500$ \\
\hline $\mathrm{XX}$ & .55 & 5.00 & 45 & 400 \\
\hline $\mathrm{X}$ & & 3.50 & 30 & 260 \\
\hline anana (Winter) & \multirow{6}{*}{\multicolumn{4}{|c|}{$\begin{array}{l}\text { Stark Delicious (Trade-Mark) } \\
\text { Stark King David (Trade-M.) } \\
\text { Stark-Star } \\
\text { Stayman Winesap } \\
\text { Wealthy } \\
\text { Wilson Red June }\end{array}$}} \\
\hline Black Ben & & & & \\
\hline Henry Clay & & & & \\
\hline Jonathan & & & & \\
\hline ady & & & & \\
\hline Rome Beauty & & & & \\
\hline
\end{tabular}

Leading Varieties

\section{Cherry}

TWO-

AXX, 5 to $7 \mathrm{ft} \ldots . .55 \mathrm{c}$

$$
\text { " } \quad \mathrm{XX}, 4 \text { to } 5 \mathrm{ft} \ldots . .50
$$

$\mathrm{X}, 3$ to $4 \mathrm{ft} \ldots . .45$

ONE-YEAR, 3 to $5 \mathrm{ft} \ldots \ldots \ldots \ldots 50$

$$
\text { " } 2 \text { to } 3 \mathrm{ft} \ldots \ldots \ldots \ldots .45
$$

Dyehouse

Early Richmond

May Duke

Montmorency King

$$
\begin{array}{lll}
\text { “ } & \text { King } & \text { Timme } \\
\text { Large } & \text { Windsor } \\
& \text { Monarch } & \text { Yellow Spanish }
\end{array}
$$

Montmorency Stark

Montmorency Sweet

$\begin{array}{rrr}10 & 100 & 1000 \\ \$ 5.00 & \$ 45 & \$ 400 \\ 4.50 & 40 & 350 \\ 4.00 & 35 & 300 \\ 4.50 & 40 & 350 \\ 4.00 & 35 & 300\end{array}$

Special Varieties

$$
\begin{array}{r}
\mathrm{XXX}, 5 \text { to } 7 \mathrm{ft} \ldots .65 \mathrm{c} \\
\mathrm{XX}, 4 \text { to } 55 \mathrm{ft} \ldots \ldots 60 \\
\mathrm{X}, 3 \text { to } 4 \mathrm{ft} \ldots \ldots 55
\end{array}
$$

$\mathrm{X}, 3$ to $4 \mathrm{ft} \ldots \ldots 5$

ONE-YEAR, 3 to $5 \mathrm{ft} \ldots \ldots \ldots \ldots 60$

Bing

$$
2 \text { to } 3 \mathrm{ft} \ldots \ldots \ldots \ldots \ldots \ldots
$$

Black Tartarian Improved

English Morello

German Ostheimer

Governor Wood

Lambert

Napoleon

$\begin{array}{rrr}10 & 100 & 1000 \\ \$ 6.00 & \$ 55 & \$ 500 \\ 5.50 & 50 & 450 \\ 5.00 & 45 & 400 \\ 5.50 & 50 & 450 \\ 5.00 & 45 & 400\end{array}$

Paul

Royal Ann

Schmidt's Bigarreau

Suda Hardy

Wragg 


\section{Peach}

Commercial Varieties

$\mathrm{XXX}, 5$ to $7 \mathrm{ft} \ldots \ldots \ldots \ldots .35 \mathrm{c}$

$\mathrm{XX}, 4$ to $5 \mathrm{ft} \ldots \ldots \ldots \ldots \ldots \ldots \ldots \ldots$

$\begin{array}{rrr}10 & 100 & 1000 \\ \$ 3.20 & \$ 28 & \$ 240 \\ 2.70 & 23 & 180 \\ 2.20 & 18 & 140\end{array}$

Alton

Arp Beauty

Banner

Belle of Georgia

Capt. Ede

Chairs' Choice

Champion

Crawford Early, Improved

Crawford Late, Improved

Early Mamie Ross

Edgemont Beauty

Engle Mammoth

Eureka:

Frances

Hiley (Early Belle)

Hyslop Cling

Illinois

\section{June Elberta}

Kalamazoo

Krummel October

Late Elberta

Levy Late Cling

Mamie Ross

Mammoth Heath Cling

Mayflower

Niagara (Mam. Crawf'd Late)

October Elberta

Red Bird Cling

Sea Eagle Improved

Stark Heath Cling

Washington (Lt. Champion)

Worth

Yellow Rareripe

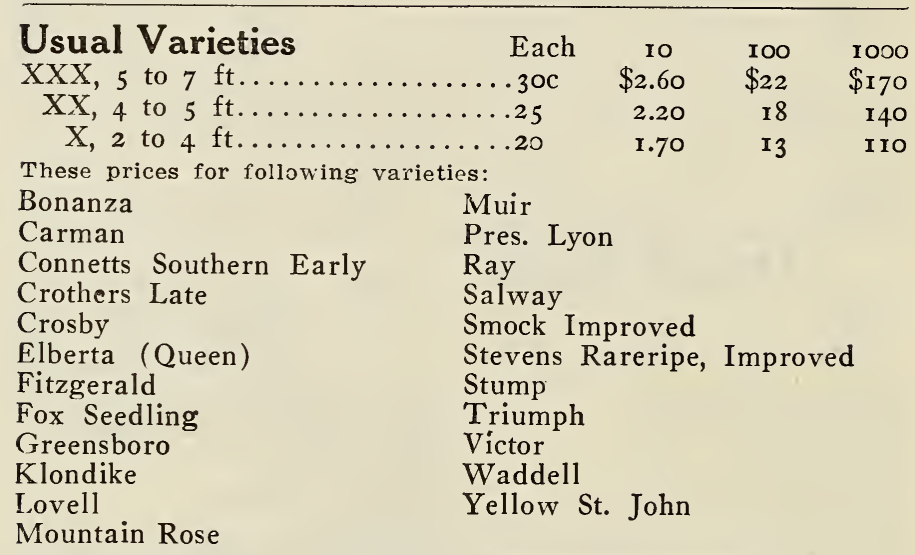

\begin{tabular}{|c|c|c|c|c|}
\hline $\begin{array}{l}\text { Special Varieties } \\
X X X, 5 \text { to } 7 \mathrm{ft} \ldots \ldots\end{array}$ & $\begin{array}{l}\text { Each } \\
.40 c\end{array}$ & $\begin{array}{c}10 \\
\$ 3.60\end{array}$ & $\begin{array}{l}100 \\
\$ 32\end{array}$ & \\
\hline $\mathrm{XX}, 3$ to $4 \mathrm{ft} . \ldots$. & .35 & 3.20 & 28 & 230 \\
\hline $\mathrm{X}, 2$ to $4 \mathrm{ft}$ & .30 & 2.60 & 22 & 180 \\
\hline
\end{tabular}

These prices for following varieties:

Elberta Cling

Gold Dust Cling

Golden Sweet Cling

Holsinger Salway

Idaho Mammoth

Marionville Cling

Martha Fern Cling
Oldmixon Free, Imp'd

Phillips Cling

Stark Summer Heath

Wallis Best

Wallis Heath Free

Yates Early Heath

\section{STARK EARLY ELBERTA}

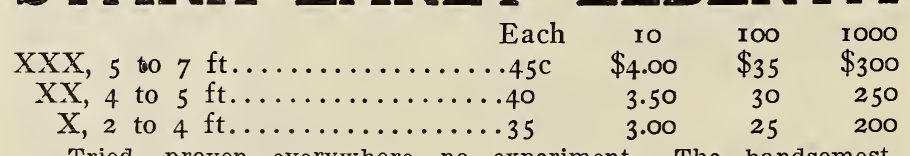

Tried, proven everywhere-no experiment. The handsomest. best, most profitable; the king of yellow peaches. Should be planted in all Elberta regions and will stand much farther north.-Stark Bros.

Ripens about ten days ahead of Elberta; the finest peach I ever tasted.-S. F. Anderson, Tuolumne Co., Calif.

Two Early Elberta trees bore more than fifty others. Ripens with Carman,-E. H. Riehl, Ill Exp. Station

My Early Elbertas have borne two crops. I don't think I ever saw finer peaches. I consider them my best peach. They ripen 10 to 15 days ahead of Elberta.-G. W. Pattee, Pinal Co., Ariz. 


\section{Pear}

Special Varieties Each

$\mathrm{XXX}, 5$ to $7 \mathrm{ft} . . . \ldots \ldots \ldots \ldots 606$

$\mathrm{XX}, 4$ to $5 \mathrm{ft} \ldots \ldots \ldots \ldots \ldots \ldots \ldots$

IO $\quad$ IOO $\quad 1000$

Anjou

Bartlett

Bartlett Hybrid

Beurre Bosc

Colorado Seedless

Comice

Crocker Bartlett

Durand

Fame (Trade-Mark)

Flemish Beauty

$\$ 5 \cdot 50$

$\$ 50$

$\$ 400$

4.50

40

360

Jones Winter

King Karl

Lincoln

Riehl Best

Stark Seckel (Riehl Strain)

Stark Tyson (Wallis Ștrain)

Theodore Williams (Stella)

Triumph

Wallis Kieffer

Winter Bartlett

Winter Nelis

Worden Seckel

\begin{tabular}{|c|c|c|c|c|}
\hline Usual Varieties & Each & Io & 100 & \\
\hline $\mathrm{XXX}, 5$ to $7 \mathrm{ft} \ldots \ldots$ & $\cdots 55 \mathrm{c}$ & $\$ 5.00$ & $\$ 45$ & $\$ 375$ \\
\hline $\mathrm{XX}, 4$ to $5 \mathrm{ft} \ldots \ldots$ & $\cdots 45$ & 4.00 & 35 & 300 \\
\hline $\mathrm{X}, 3$ to $4 \mathrm{ft}$. & $\cdots 35$ & 3.00 & 25 & 200 \\
\hline Alamo & Kieffer & & & \\
\hline Bloodgood & Koonce & & & \\
\hline Boussock & Lawrence & & & \\
\hline Clairgeau & Manning & Elizal & & \\
\hline Clapp Favorite & Patrick I & arry & & \\
\hline Dorset & Rossney & & & \\
\hline Duchess (Angouleme) & Snyder & & & \\
\hline Garber & Vermont & Beauty & & \\
\hline Howell & Wilder $\mathrm{F}$ & arly & & \\
\hline
\end{tabular}

\section{Plum}

Special Varieties Each to too 1000

XXX, 5 to $7 \mathrm{ft} \ldots \ldots \ldots \ldots \ldots \ldots . \cdots 5 \mathrm{c}$ \$5.00 $\$ 45$ \$400

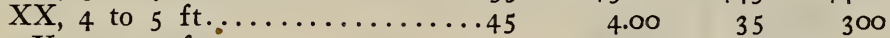

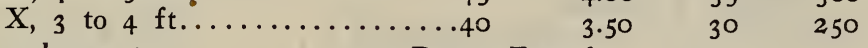

Abundance

Prune French

America

Burbank

Early Gold (Shiro)

Freestone Goose

October Purple

Omaha

Purple Flesh

Gold (Trade-mark)

Late Goose

Red June

Satsuma

Stella

Stark Green Gage (Missouri) Tatge (Imp'd Lombard)

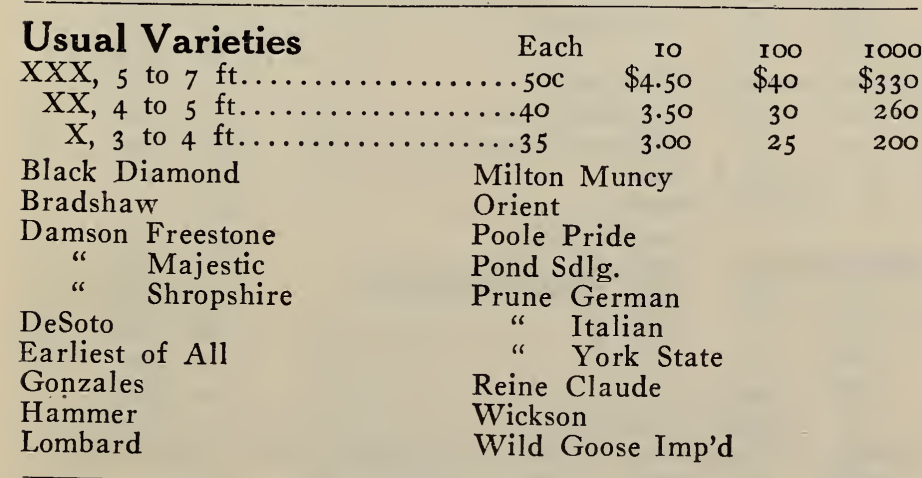

\begin{tabular}{|c|c|c|c|}
\hline DUARTE: & Each & IO & 100 \\
\hline $\mathrm{XXX}, 5$ to $7 \mathrm{ft} \ldots \ldots$ & . . 9оc & $\$ 8.50$ & $\$ 80$ \\
\hline$X X, 4$ to $5 \mathrm{ft} .$. & .80 & 7.50 & 70 \\
\hline
\end{tabular}




\section{Dwarf Pear}

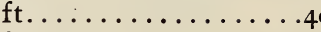

Anjou

3 to $4 \mathrm{ft} \ldots \ldots \ldots \ldots \ldots \ldots \ldots$

Bartlett

Clapp Favorite

Howell

Comice

Duchess

King Karl

Manning Elizabeth

Stark Seckel

Winter Bartlett

Fame:

Triumph

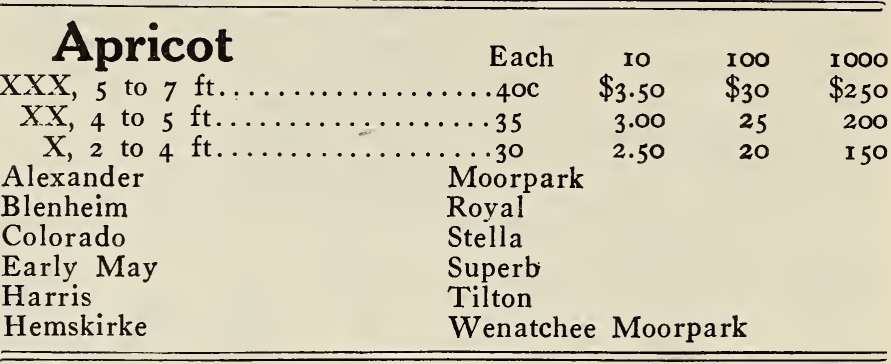

\section{Quince}

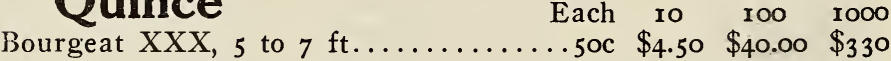

" XX, 4 to $5 \mathrm{ft} \ldots \ldots \ldots \ldots \ldots 40 \quad 3.50 \quad 30.00 \quad 250$

" $\quad \mathrm{X}, 2$ to $4 \mathrm{ft} \ldots \ldots \ldots \ldots \ldots . \ldots 30 \quad 2.50 \quad 22.00 \quad 180$

Mo. Mammoth XXX, 5 to $7 \mathrm{ft} \ldots \ldots \ldots \ldots 45 \quad 4.00 \quad 35.00 \quad 280$

" " $"$ XX, 4 to $5 \mathrm{ft} \ldots \ldots \ldots \ldots 35 \quad 3.00 \quad 25.00200$

$\mathrm{X}, 2$ to $4 \mathrm{ft} \ldots \ldots \ldots .25 \quad 2.20 \quad 17.00 \quad \mathrm{I} 30$

Orange XXX, 5 to $7 \mathrm{ft} \ldots \ldots \ldots \ldots \ldots \ldots . \ldots \ldots+\ldots \ldots$

" $\mathrm{XX}, 4$ to $5 \mathrm{ft} \ldots \ldots \ldots \ldots \ldots \ldots 40 \quad 3.50 \quad 30.00 \quad 250$

$\mathrm{X}, 2$ to $4 \mathrm{ft} \ldots \ldots \ldots \ldots \ldots \ldots$......... $2.50 \quad 22.00 \quad 180$

Van Deman' (Tr.-mark) XXX, 5 to $7 \mathrm{ft} .65$ 6.00 $57.00 \quad 470$

" " " " $" \mathrm{XX}, 4$ to $5 \mathrm{ft} .65 \quad 5.00 \quad 47.00 \quad 370$

" " " " $\quad \mathrm{X}, 2$ to $4 \mathrm{ft} .45 \quad 4.00 \quad 37.00 \quad 280$

\section{Nut Trees}

Chestnut, American Sweet 3 to $4 \mathrm{ft} \ldots \ldots \ldots$ Each ro 100

4 to $6 \mathrm{ft} . \ldots \ldots \ldots \ldots 45 \quad 4.20 \quad \$ \ldots$

" Grafted, Rochester (fr. E. H. Riehl) $\$$ r.oo $\ldots . . .$.

" " Cooper (another of Riehl's) r.oo $\ldots . . \ldots$

English Walnut, Select Seedlings......... $40 \quad 3.70 \quad 30$

Concord Seedlings ........ 50 . $4.70 \quad \ldots$

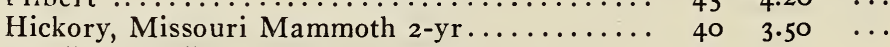

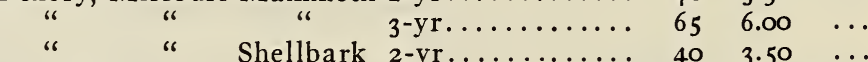

" " $\quad$ " $\quad$ "yr.......... 65 6.00 6.

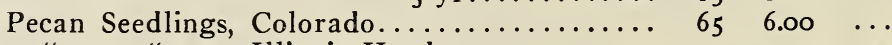

" " Illinois Hardy............ $45 \quad 4.20 \quad 35$

Missouri Hardy......... $45 \quad 4.20 \quad 35$

Grafted Pabst................ r.25 ... ...

" Stuart $\ldots \ldots \ldots \ldots \ldots \ldots \ldots \ldots \ldots$ r.25 $\ldots \ldots \ldots \ldots$

" " Van Deman ............ 1.25 .......

Walnut, Black r to $2 \mathrm{ft} . \ldots \ldots \ldots \ldots \ldots \ldots \ldots$ ro 8 o 6

" " 2 to $3 \mathrm{ft} . \ldots \ldots \ldots \ldots \ldots \ldots \ldots \ldots$ I5 1.20 10

" " $\quad 3$ to $4 \mathrm{ft} \ldots \ldots \ldots \ldots \ldots \ldots \ldots \ldots \ldots$ 20 1.80 16

" " 4 to $6 \mathrm{ft} . \ldots \ldots \ldots \ldots \ldots \ldots \ldots \ldots 25 \quad 2.20 \quad 18$

" Japan .................... 40 $3.50 \quad \ldots$

"White (Butternut) ............ 65 6.00 6.

Forest Tree Seedlings Each 1000000

Catalpa Speciosa, r to $2 \mathrm{ft} \ldots \ldots \ldots \ldots \ldots \ldots \ldots \ldots \ldots 2.00 \$ \mathrm{r}_{3}$

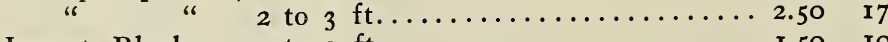

Locust, Black I to $2 \mathrm{ft} \ldots \ldots \ldots \ldots \ldots \ldots \ldots \ldots \ldots$ r.50 ro

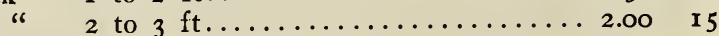


25 of one variety, Grape, Raspberry, Blackberry, Dewberry, Currant, Gooseberry, Roses, Shrubs, Climbers, Hedge Plants, Garden Roots, Paeonies, Ornamental Trees at 100 rate; 300 at 1,000 rate.

\begin{tabular}{|c|c|c|c|c|c|}
\hline \multicolumn{3}{|c|}{ TWO-YEAR } & \multicolumn{3}{|c|}{ ONE-YEAR } \\
\hline Eac & & & Each & 100 & 1000 \\
\hline I $5 \mathrm{C}$ & $\$ 9.00$ & $\$ 60$ & Agawam (d. red) rich and sweet. Ioc & $\$ 6.00$ & $\$ 40$ \\
\hline 40 & 35.00 & ... & Banner (Trade-mark) rich red. 30 & 25.00 & \\
\hline 40 & 35.00 & & Barry (black) high quality......30 & 25.00 & \\
\hline 20 & 12.00 & 80 & Brighton (red) one of the best.. I 5 & 8.00 & \\
\hline 25 & 20.00 & I 50 & Campbell Early (blk) very large.20 & I 5.00 & I IC \\
\hline I 5 & 10.00 & 70 & Catawba (red) an old favorite.. Io & 7.00 & \\
\hline I 5 & 7.00 & 35 & Clinton (black) fine for wine... ro & & \\
\hline 15 & 7.00 & 35 & Concord (blk) the money-maker. Io & & \\
\hline 20 & 14.00 & 100 & Delaware (red) small, best qual.is & 12. & \\
\hline 20 & 12.00 & 80 & Diamond (white) early, hardy.. I 5 & & \\
\hline 40 & 35.00 & ‥ & rly Daisy (black)............ & $25 \cdot 0$ & \\
\hline 20 & 12.00 & 80 & Early Ohio (black) early....... I5 & 8 & \\
\hline 30 & 25.00 & .. & Goethe (red) best in quality ....25 & 20.00 & \\
\hline 35 & 30.00 & 250 & Green Mountain (white) excel..25 & 20.00 & \\
\hline 40 & 35.00 & 300 & for table...30 & 25 . & \\
\hline 25 & OO & I IO & oductive. 20 & $14 \cdot$ & \\
\hline I 5 & 9.00 & 60 & Ith...... Io & 5 & 3 \\
\hline 25 & 20.00 & I6o & King (black) mam. Concord...20 & 15.00 & \\
\hline 30 & 25.00 & 200 & Lucile (red) beautiful and good.25 & 20. & \\
\hline 20 & & IOO & ependable.... I 5 & 9.00 & \\
\hline I 5 & ০o & 55 & Lindley (red) keeps and ships.. Io & 5.00 & \\
\hline 20 & I 5.00 & 80 & Moore Early (black) valuable..I5 & 10.00 & 5 \\
\hline 20 & I2. & 70 & ra (white) most popular..I 5 & 8.00 & 4 \\
\hline 35 & 28.00 & 240 & rior for wine.25 & 20.00 & I 6 \\
\hline 35 & 30.00 & $\cdots$ & 1 (red) good quality; hardy.25 & 20.00 & \\
\hline I5 & 7.00 & 50 & Salem (red) for table and wine.. ro & 5.00 & 3 \\
\hline 75 & 65.00 & . & Stark Delicious (T-mark) (red) ..6o & 50.00 & . \\
\hline 30 & 25.00 & 200 & Stark Eclipse (T-mark) (blk.) .25 & 20.00 & I 5 \\
\hline 50 & 40.00 & 300 & Stark King Philip (T.Mk.) (blk.) 40 & 30.00 & 200 \\
\hline I5 & 10.00 & 65 & fair quality.... Io & 5.00 & $3 c$ \\
\hline 25 & 20.00 & I 50 & ler (black) sweet and rich.20 & I 5.00 & 100 \\
\hline 25 & 17.00 & 125 & Woodruff (red) handsome, showy.20 & 13.00 & 9 \\
\hline 20 & 12.00 & 80 & Worden (black) large and fine... I5 & 8.00 & \\
\hline
\end{tabular}

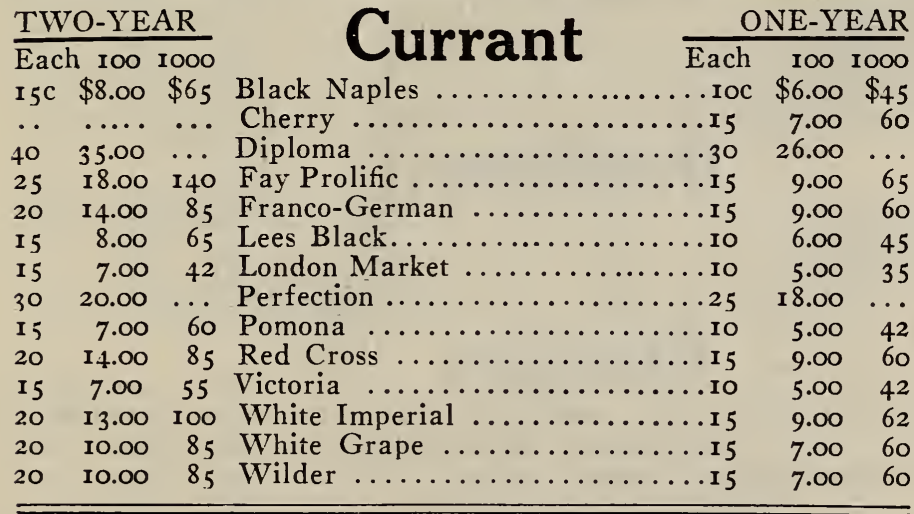

TWO-YEAR

Each 100 1000 $40 \mathrm{OC} \$ 30.00 \$$.. 35

40

20

I 5

20

40

I 5

20

20

40

I 5

\section{Gooseberry}

Crown Bob

$30.00 \ldots$ Chautauqua $\ldots \ldots \ldots \ldots \ldots \ldots \ldots$

$35.00 \ldots$ Carman .......................

I 2.0090 Downing .............. 15

$8.00 \quad 65$ Houghton ................

$12.00 \ldots$ Josselyn ...................

$30.00 \ldots$ Lancashire ..................

II.00 ... Mountain ................

I6.00 I ro Oregon Champion ............ I5

I6.00 ... Pearl .................. I5

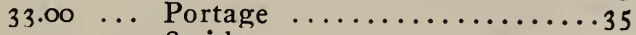

I0.00 ... Smith ................

ONE-YEAR

$$
\begin{array}{rr}
\$ 25.00 & \$ \ldots \\
25.00 & \ldots \\
25.00 & \ldots \\
9.00 & 75 \\
7.00 & 50 \\
8.00 & \ldots \\
25.00 & \ldots \\
7.00 & \ldots \\
12.00 & 90 \\
12.00 & \ldots \\
25.00 & \ldots \\
7.00 & \ldots
\end{array}
$$$$
001000
$$ 


\section{Ornamental Trees}

$\begin{array}{ccc}\text { Each } & 10 & 100 \\ 25 \mathrm{C} & \$ 2.20 & \$ 15\end{array}$ $\ldots \ldots \ldots \ldots 3$ to $5 \mathrm{ft}$. $30 \quad 2.6020$ $\ldots \ldots \ldots \ldots 5$ to $7 \mathrm{ft}$. $40 \quad 3.60 \quad 25$

Birch, Cut-Leaf Weeping........ 3 to $5 \mathrm{ft}$. 50 4.50 ..

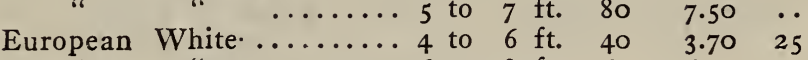
$6.00 \quad 45$ $9.00 \quad \ldots$ I3.00 4.50 . $5 \cdot 50 \quad$ $5 \cdot 50$ 6.50 3.60 . $4.50 \quad 35$ 6.5060 9.00 .. $4.50 \quad-$ $5 \cdot 50 \quad$ $3.20 \quad 22$ $4.20 \quad 28$ $6.00 \quad$. $4.50 \quad 28$ $5.50 \quad 35$ II.00 80 I 3.00100 I. 80 I 2 $3.20 \quad 20$ $4.70 \quad 35$ $7 \cdot 50 \quad 60$ $3.70 \quad 30$ $4.50 \quad 40$ $6.50 \quad 60$ 4.20 .. $5.50 \quad \ldots$ $3.20 \quad 25$ $4.50 \quad 30$ $3.20 \quad 25$ $5.00 \quad 35$ $7 \cdot 50$ 9.00 $3.70 \quad \ldots$ $5.70 \quad$.. $3.20 \quad 25$ $5.00 \quad 35$ $2.50 \quad 20$ $7.50 \quad \ldots$ $3.20 \quad 25$ $5.00 \quad 30$ 4.00 . $4.00 \quad \ldots$ $4.00 \quad \ldots$ 5.00 . $5.50 \quad \ldots$ $7.00 \quad$.. $2.20 \quad 15$ $2.80 \quad 30$ $5.50 \quad 40$ 9.0075

I 1.00 . $2.20 \quad$ I 5 $3.80 \quad 30$ $5.50 \quad 40$ $9.00 \quad 75$ II.00 - . $3.20 \quad 25$ $4.50 \quad 35$ 


\section{Ornamental Trees---Con'd.}

Poplar, Norway............. I2 to I5 ft. 1.50

Persimmon ............... 3 to $4 \mathrm{ft}$. 50 $\ldots \ldots \ldots \ldots \ldots+\ldots \ldots$ to $5 \mathrm{ft}$. 60

Russian Olive.............. 2 to $3 \mathrm{ft}$. 30

Sycamore, American..........4 to $6 \mathrm{ft}$. 35

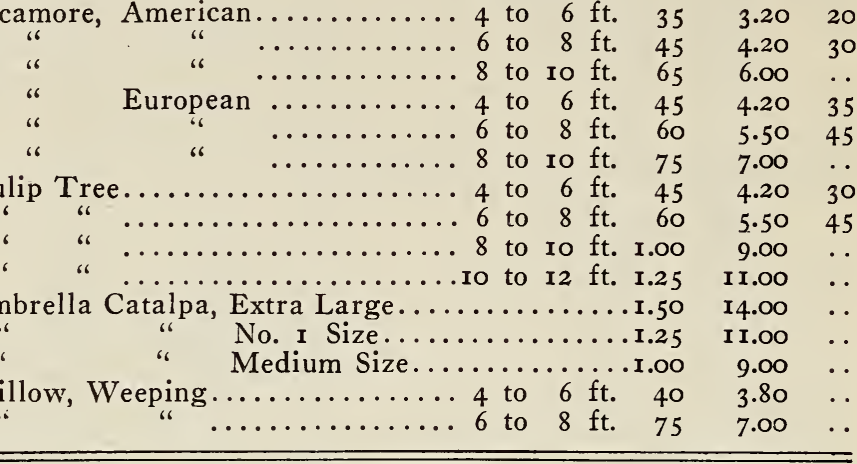

\section{Roses} ....35c $\$ 25.00 \$ 180$ Baltimore Belle (Climber). Clusters of blos..25 I8.00 I20 Burbank. Cherry rose colored blossoms.......50 $40.00 \quad 300$ Caroline Testout. Clear, beautiful pink.......50 40.00 ... Clio. Delicate flesh color .................40 $30.00 \quad \ldots$ Conrad F. Meyer. Beautiful silvery rose.....50 $40.00 \quad \ldots$ Crimson Rambler. Solid mass of crimson bty..35 25.00 i 80 Dorothy Perkins (Climber). Clear shining pink.35 $25.00 \quad \ldots$ Eugene Furst. Very dark red.............. 40 40.00 ... Everblooming Crimson Rambler............75 65.00 ... General Jacqueminot. Rich velvety crimson...50 40.00 300 Gruss an Teplitz, Reddest of all red roses....50 40.00 300 Helen Gould. Shaded dark carmine.........50 40.00 300 Hugh Dixon. Beautiful brilliant crimson......50 40.00 300 J. B. Clark. A dazzling crimson............50 $40.00 \quad 300$ Jessie. (Improved Baby Rambler) ..........40 $30.00 \quad 200$ Lady Gay (Climber). Deep, rich rose pink...440 30.00 ... Madame Plantier. Requires no protection....30 20.00 I 60 Marshall P. Wilder. Dark cherry red......4. $30.00 \quad \ldots$ Mrs. John Laing. Splendid satiny pink......40 $30.00 \quad \ldots$ Paul Neyron. Largest, most glorious rose.....35 $25.00 \quad$ I80 Pink Moss. Hardy, rugged, well known.....25 I8.00 I30 Queen of Prairies. Bright rosy red........25 I8.00 120 Red Moss. Best and handsomest moss......25 I8.00 I30 Rosa Rugosa (Japan. Rose). Glorious light red.40 $30.00 \quad \ldots$ Rosa Rugosa Alba. Handsome single ẉhite...40 $30.00 \quad \ldots$ Rubin (Climber). Large scarlet blossoms....40 $30.00 \quad \ldots$ Snow Queen. The white American Beauty....50 40.00 ... Trier (Climber). Creamy white, semi-double..40 $30.00 \quad 200$ Ulrich Brunner. Rich, flashing crimson.......50 $40.00 \quad 300$ White Dorothy (Climber). Very handsome....50 40.00 … Wm. R. Smith. Handsome salmon pink.......50 $40.00 \quad \ldots$

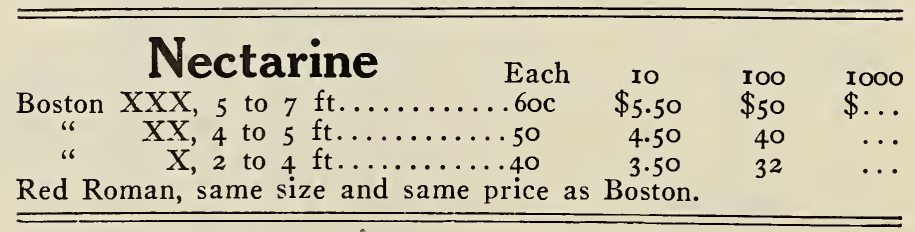

Stark Delicious finest quality known, beautiful, hangs on during dry weather. A long keeper, best commercial variety, stands handling well. Best apple in the world.-Jno. Cottle, Washington Co., Ohio.

Stark Delicious the best quality apple grown. My nine-yearold trees have borne four crops; the best money-maker.-H. D. Morton, Pope Co., Ark. 


\section{Ornamental Shrubs}

Althea Seedlings .................... $\$ 17.00 \$ \ldots$

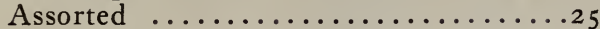
$17.00 \quad \ldots$

Aralia Pentaphylla (Angelica Tree)........40 $33.00 \quad 250$

Calycanthus Floridus (Carolina Allspice)...40 $33.00 \quad \ldots$

Cercis Canadensis (Judas Tree or Redbud) ...30 $22.00 \quad \ldots$

Chionanthus Virginica (Fringe Tree).......40 $33.00 \quad \ldots$

Cornus Florida (Dogwood)................. 255 .

Cydonia Japonica (Scarlet Japan Quince) ....35 $25.00 \quad \ldots$

Forsythia Suspensa ..................... 255 .

Halesia Tetraptera (Snowdrop or Silver Bell).40 $33.00 \quad \ldots$

Hamamelis Virginica (Witch Hazel).......30 $22.00 \quad \ldots$

Hibiscus Mehanii (Lavender Rose of Sharon).40 $33.00 \quad \ldots$

Totus Alba (White Rose of Sharon). $40 \quad 33.00 \quad \ldots$

Hydrangea Arborescens Sterilis (Hills of Snow) $40 \quad 33.00 \quad \ldots$

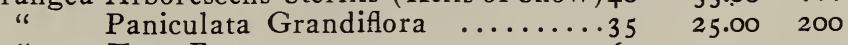

" Tree Form .................60 55.00 ...

Lilac Common Purple...................20 $\quad$ I5.00 II

Common White ....................

Budded, Belle de Nancy.............40 $33.00 \quad \ldots$

" Frau Dammann ..........40 $33.00 \quad \ldots$

" Henri Sladek (Imp. L. Spath).90 $80.00 \quad \ldots$

" Ludwig Spath ............40 $33.00 \quad \ldots$

" Michael Buchner ...........40 $33.00 \quad \ldots$

“ Madame Abel Chatenay.......40 $33.00 \quad \ldots$

“ “ " $\quad$ President Grevy ............40 $33.00 \quad \ldots$

" Tartarica Alba (White Tartarian).40 $33.00 \quad \ldots$

“ Tartarica Grand. (Pink).........40 33.00 ...

Philadelphus Coronarius (Mock Orange) $\ldots 25$ I $8.00 \quad$ I 50

$20.00 \quad 170$

$33.00 \quad 260$

$25.00 \quad 200$

$22.00 \quad 180$

$25.00 \quad 200$

$25.00 \quad \ldots$

$33.00 \quad \ldots$

$18.00 \quad I 50$

$33.00 \quad 250$

I $6.00 \quad 120$

$33.00 \quad 250$

$33.00 \quad \ldots$

$33.00 \quad \ldots$

$16.00 \quad 120$

$25.00 \quad \ldots$

$25.00 \quad 200$

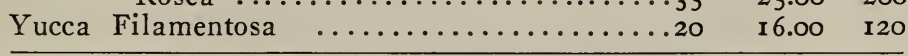

\section{Garden Roots}

Asparagus, Bonvallette's Giant............... \$r.50 \$ ro

" Conover's Colossal .............

" Mammoth White ............. 1.50 ro

" Palmetto ................... 1.50 10

Rhubarb, Myatt's Linnaeus ............. $15 \quad 5.00 \quad 35$

" Victoria Giant ................. 15 $8.00 \quad 50$

Horseradish $\ldots \ldots \ldots \ldots \ldots \ldots \ldots \ldots \ldots \ldots$ ro $5.00 \quad 30$

\section{Hedge Plants}

Each 100 1000

Barberry, Japanese ...................20 \$1 $\$ 5.00$ \$120

Japan Quince ............. I8 to 24 inch 20 15.00 120

Privet, Amoor River (North) ...I 8 to 24 inch 20 I 5.00 I2O

" " " $" \ldots \ldots \ldots \ldots, 2$ to 3 feet $25 \quad 20.00 \quad 150$

California ..........18 to 24 inch ro $7.00 \quad 45$

" $\quad \ldots \ldots \ldots \ldots, 2$ to 3 feet $12 \quad 8.00 \quad 55$

Ibota $\ldots \ldots \ldots \ldots \ldots \ldots \ldots$ I 8 to 24 inch $1_{5} \quad 12.00 \quad 65$

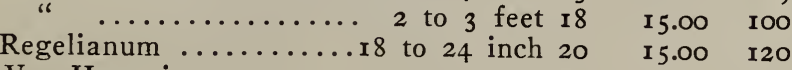

Spirea, Van Houttei...................20 I6.00 $\quad$ I30 


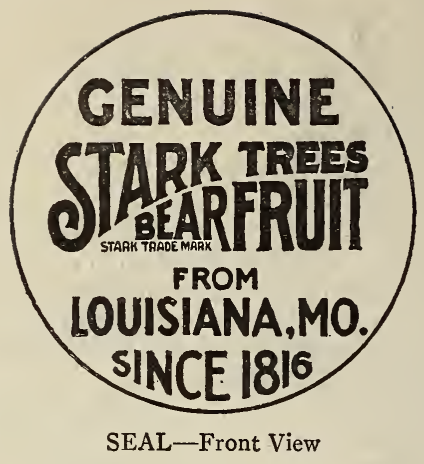

F VERY bundle, bale, box or car of genuine Stark Trees is locksealed with this trade-mark seal. This is a guarantee to the planter that he is getting the best nursery stock grown in America - Stark Trees.

Behind this seal is the reputation of the largest, most up-to-date nursery organization in the world. Stark Sealed Trees are the result of four generations of tree-growing experience-since 1816 .

Do not overlook the fact that genuine Stark Trees can be bought only at Louisiana, Mo.

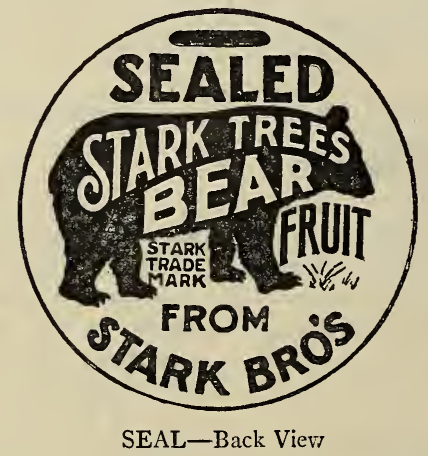

